\title{
Calculation of a Radial Inhomogeneous Rock Mass with a Spherical Cavity under the Influence of Thermal Field
}

\author{
Irina I. Frolova ${ }^{1, a}$ \\ ${ }^{1}$ National Research Moscow State University of Civil Engineering, 26 Yaroslavskoye Shosse, \\ Moscow, 129337, Russia \\ ifrolova51@inbox.ru
}

Keywords: radial inhomogeneity, thermal field, rock mass, spherical cavity, numerical analytic solution.

\begin{abstract}
The problem considered is that of calculating the stress-strain behavior of a rock mass in the vicinity of a spherical cavity, taking into account the radial inhomogeneity of the rock mass material. The solution takes into account the technological inhomogeneity of the soil and the inhomogeneity caused by the influence of the thermal field. The axially symmetric problem of determining the stresses and displacements was solved with a joint action of an asymmetric load (ground reaction) and hyper-thermal fields on the rock mass. Similar problems tend to arise during the creation of underground cavities and their exploitation for storing various self-heating products. To solve the initial simultaneous equations in displacements, the Fourier series of Legendre polynomials were used. Then by the separation of variables the problem was reduced to 2 ordinary differential simultaneous equations with variable coefficients, which can be solved numerically.
\end{abstract}

\section{Introduction.}

One of the objective properties of almost all materials is their inhomogeneity, both natural and technological, which appears in the establishment and operation of facilities. Therefore, a significant number of modern studies are approaching the solution of the problems posed from the standpoint of the theory of elasticity of inhomogeneous bodies. In addition, due to the extensive development of both theoretical and experimental methods, using the capabilities of modern computer technology, it became possible to solve many important practical problems in which the inhomogeneity of the materials is a characteristic feature.

\section{Algorithm of the calculation.}

The present study considers the solution of the axially symmetric problem of determining the stresses and displacements that arise when the rock mass is jointly influenced by an asymmetric load (ground reaction) and a centrally symmetric thermal field. The calculation model of the problem is shown in Fig.1. The subject is a rock mass containing a spherical cavity, created by an explosion. A nuclear explosion creating an underground cavity almost instantaneously releases in this cavity a large amount of heat and causes a considerable heating of the rock mass in the proximity of the cavity, followed by the heat transmission into the interior of the rock mass and the cooling of the cavity surface. This is a nonstationary process, but excluding from consideration the initial period and taking into account the relatively slow redistribution of the thermal field [1] during the subsequent sufficiently long time, the problem of determining the thermal stresses can be considered as quasi-stationary one [2,3].

Another problem, related to the calculation of thermal stresses, considers the continuous heat flux e.g. conditioned by the storage of self-heating products in the cavity. In this case, a constant temperature will be maintained on the cavity surface. In both cases, the corresponding thermal fields cause a change in the physical and mechanical characteristics of the material of the rock mass $[4,5]$. 


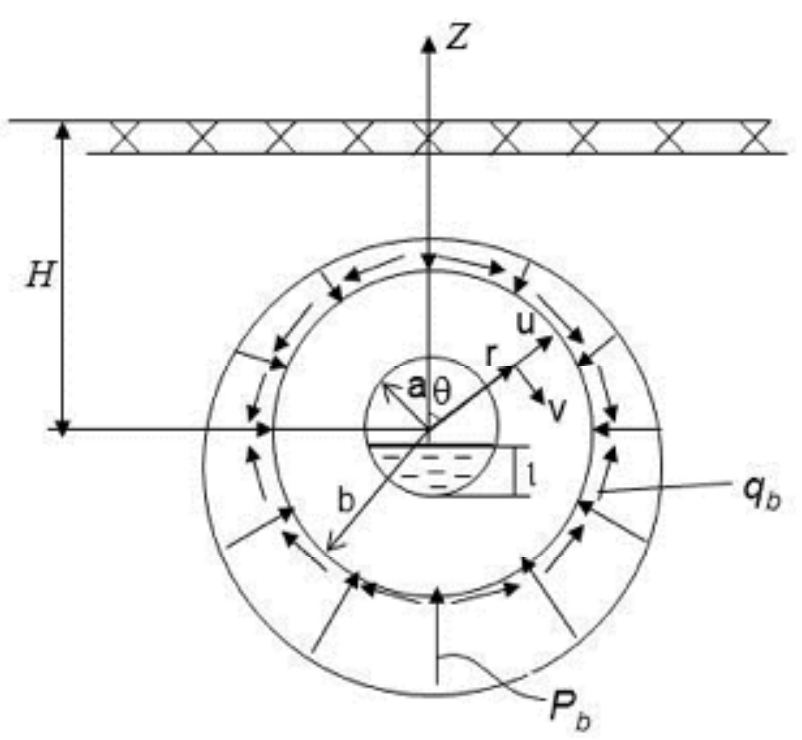

Fig.1. The calculation scheme of the rock massif

As the problem was solved in a stationary formulation, then a characteristic as elastic as $E(r)$, and the related coefficients of $\lambda(r), \mu(r)$ and $k(r)$ depend only on one coordinate: the radius $r$.

The calculation took into account the actual conditions of the rock mass (the presence of ground reaction, the sole weight of the rock mass) and the dependence of the soil's mechanical characteristics on both temperature and rock jointing of the mass [6,7].

$$
E(r)=E_{0} e^{-\delta T}\left[1+\left(k_{1}-1\right)\left(\frac{a}{r}\right)^{m}\right]
$$

where: $k_{1}$ and $m$ - empirical coefficients, $r$ - cavity radius, $E_{0}$ - modulus of elasticity value if $T=0^{\circ} \mathrm{C}, \delta-$ empirical parameter.

A numerical analytic solution is applied for solving the class of problems on the equilibrium of elastic inhomogeneous bodies in spherical coordinates. An axially symmetric problem of this class can be reduced to a system of two partial differential equations with variable coefficients relative to the displacement components of $u_{n}$ and $v_{\mathrm{n}}[8,9]$. The solution of the derived equations is proposed to be sought in the form of an expansion into Fourier series in Legendre polynomials [10]. As a result, a system of two ordinary differential equations of second order with variable coefficients can be obtained for the $n$-th expansion term. The algorithm for numerical implementation of the problem consists in reducing the derived simultaneous equations for each member of the Fourier series to the systems of first-order differential equations, whose further solution is carried out by the matrix orthogonal sweep method, described in detail in [11].

\section{Results of the calculation.}

The calculations on the basis of the developed program were made with the following initial data: $a=25 \mathrm{~m}, b=250 \mathrm{~m}, H=1200 \mathrm{~m}, \gamma=2.1510^{3} \mathrm{~kg} / \mathrm{m}^{3}, \mathrm{E}_{0}=210^{4} \mathrm{MPa}, \mathrm{v}=0.23$.

A stationary problem considered below: thermal field with the temperature $T_{0}$ constantly maintained on the contour of a cavity with a radius $r=a$, and the temperature $T_{1}$ maintained at a considerable distance from the cavity.

In the case of a stationary regime, the following temperature distribution takes place in the rock mass:

$$
T(r)=\frac{\left(T_{0}-T_{1}\right) a b}{r(b-a)}+\frac{T_{1} b-T_{0} a}{(b-a)}
$$

With a stationary thermal field, the rock mass is heated to a considerable depth, which is explained by the duration of thermal field influence. In this case, the calculation of stresses caused 
only by the thermal influence, without taking into account the force loads $\left(p_{b}=0, q_{b}=0\right)$ is of considerable interest. The calculation was carried out for two thermal differentials $\left(T_{0}=60^{\circ}\right.$ and $T_{1}=20^{\circ}$, and $T_{0}=120^{\circ}$ и $T_{1}=40^{\circ}$ ). In this case, the inhomogeneity caused by only rock jointing of the rock mass $(\delta=0)$ was taken into consideration. Fig.2 shows that taking into account the inhomogeneity of the rock mass material $\left(k_{l}=0.5\right)$ leads to a significant decrease in the level of thermal stresses $\sigma_{\theta}{ }^{\mathrm{T}}$, especially on the cavity contour, where this difference approximates to $40 \%$, and almost completely disappears if $r>3 a$. The same graphs show that calculating only the thermal components of the stresses along the radius $r$ at $\theta=90^{\circ}$, we have both a compression zone (near the cavity) and a stretching zone for $r>4 a$.

Fig. 2. Power $\sigma_{\theta}{ }^{\mathrm{P}}$ and temperature $\sigma_{\theta}{ }^{\mathrm{T}}, \sigma_{\mathrm{r}}{ }^{\mathrm{T}}$ stresses at a stationary temperature field

The difference in stresses,$\sigma_{\mathrm{r}}^{\mathrm{T}}$ while taking the inhomogeneity into account begins to affect the rock mass at some distance from the cavity, at $r=2 a$, but also almost completely disappears at $r>3 a$.

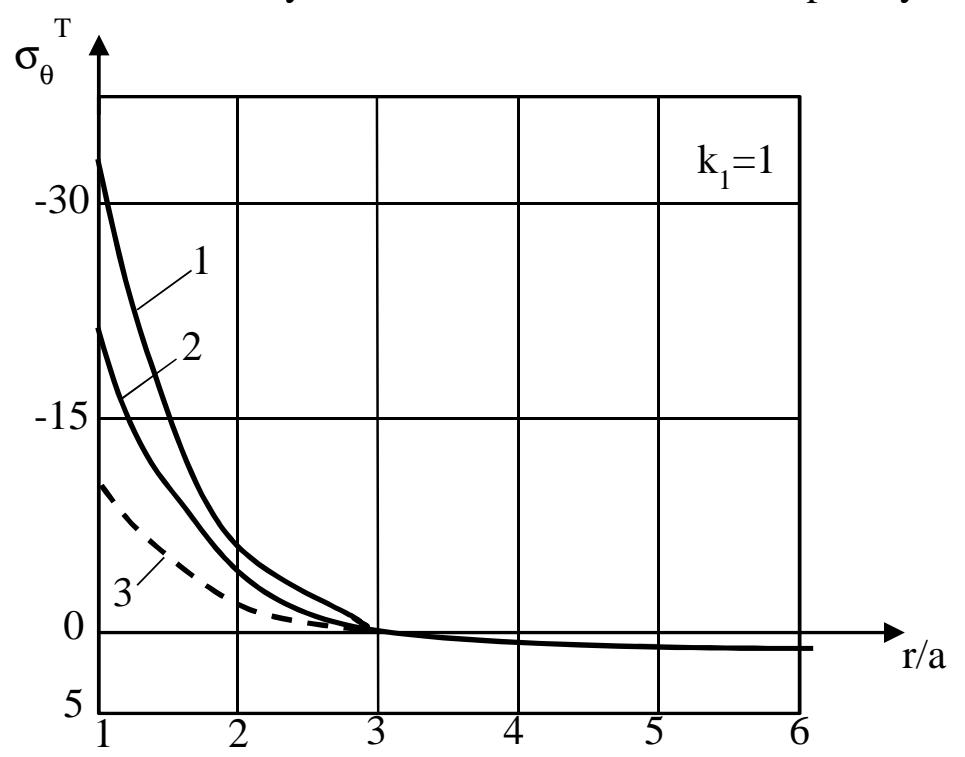

Fig.3 Influence of the parameter $\delta$ on the temperature stress $\sigma_{\theta}{ }^{\mathrm{T}}$. $1-\delta=0 ; 2-\delta=0.01\left(\Delta \mathrm{T}=40^{0}\right) ; 3-\delta=0.01\left(\Delta \mathrm{T}=80^{0}\right)$.

The comparison of the stress level caused by force loads with the stress level caused by the thermal field also proves interesting. Besides, Fig. 2 shows the stress diagrams $\sigma_{\theta}{ }^{\mathrm{P}}$ at $\mathrm{H}=1200 \mathrm{~m}$, $\gamma=2.1510^{3} \mathrm{~kg} / \mathrm{m}^{3}$, and also the strain $\sigma_{\theta}^{\mathrm{T}}$ at $\Delta \mathrm{T}=80^{0}, \alpha=1.510^{-5} 1 / \mathrm{deg}, \mathrm{E}_{0}=210^{4} \mathrm{MPa}$. It can be seen that the stresses caused by these two kinds of loads are of the same order. It is obvious that a change in $\mathrm{H}$ or a change in the thermal differential $\Delta \mathrm{T}$ can lead to a redistribution of these diagrams.

Testing this task, the thermal stresses and the stresses caused only by the force loads were calculated separately. The obtained results were summed up, which could be carried out at $\delta=0$. The stresses obtained with simultaneous consideration of both influences are in complete accord with the total stresses.

During the further investigation of the influence of the rock mass inhomogeneity on the stress state, special attention was paid to the influence of the $\delta$ parameter, i.e. account for the inhomogeneity associated with the thermal effect. Fig. 3 shows the diagrams $\sigma_{\theta}{ }^{\mathrm{T}}$ calculated at the different values of the $\delta$ parameter with the thermal differential of $\Delta \mathrm{T}=40^{\circ}$. Taking inhomogeneity into account has a visible effect on the values of the thermal stresses. The same figure shows a dotted curve of $\sigma_{\theta}{ }^{\mathrm{T}}$ at $\delta=0.01$ and $\Delta \mathrm{T}=80^{\circ} \mathrm{C}$. In the case of homogeneous structure $(\delta=0)$, the 
diagrams obviously coincide for all values of $\Delta \mathrm{T}$. The difference in the stress values is conditioned by the significantly greater influence of temperature inhomogeneity at higher temperatures.

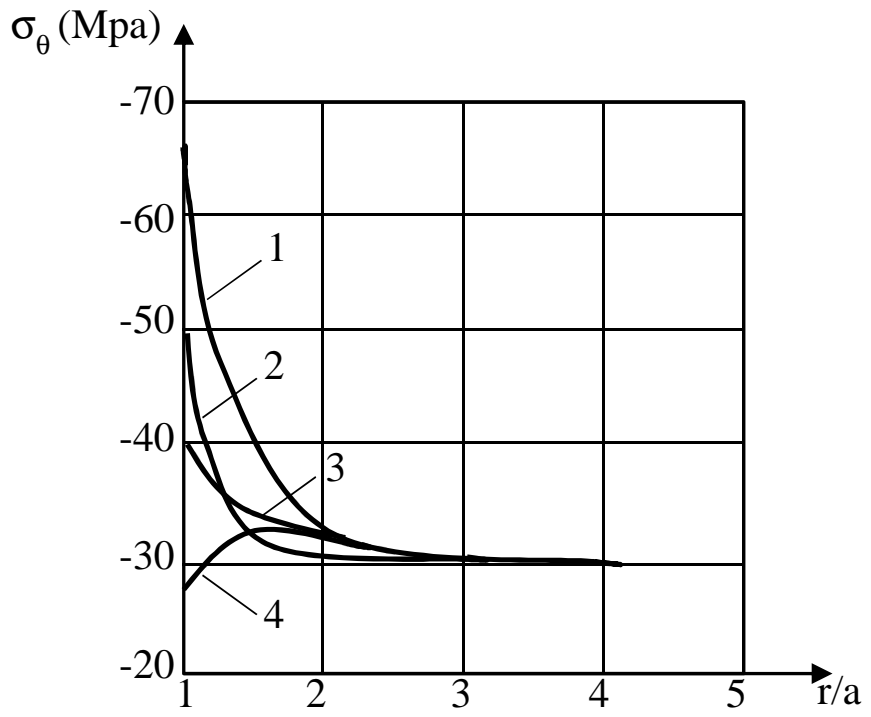

Fig.4 Change of total stresses $\sigma_{\theta}$ along the radius $\left(\theta=90^{\circ}\right)$. $1-\mathrm{k}_{1}=1, \delta=0 ; 2-\mathrm{k}_{1}=1, \delta=0.01 ; 3-\mathrm{k}_{1}=0.5, \delta=0 ; 4-\mathrm{k}_{1}=0.5, \delta=0.01$.

\section{Conclusions}

In the conclusion of this study, it's worth noting that the results shown in Fig.4 (diagrams of total stresses for homogeneous and inhomogeneous materials) allow to assert that at $\delta>0$, there is a decrease not only in the thermal stresses but also in the stresses caused by the ground reaction. Thus, the inhomogeneity of the rock mass significantly affects the results obtained in solving problems about bodies that include the real conditions for their creation and exploitation.

\section{References}

[1] A.A. Bakirov, Application of underground nuclear explosions in the oil industry, Nedra, Moscow, 1981.

[2] I.I. Frolova, Non-stationary problem of thermal stress state of inhomogeneous array with a spherical cavity, Scientific Review, 12 (2015) 94-99.

[3] Frolova I.I. Calculation of the non-stationary temperature field in an inhomogeneous massif with a spherical cavity filled with a heated liquid. Scientific review. 2016. №9. c.12-16.

[4] Andreev V.I., Avershyev A.S. The stress state in the rock mass exposure to moisture and temperature fields. Procedia Engineering vol. 111 (2015), pp. 30-35.

[5] Andreev V.I, Bulushev S.V. Optimization of an inhomogeneous thick-walled spherical shell in a temperature field. Bulletin of MGSU. 2012. N12. pp. 40-46.

[6] Meschyan S.R. Mechanical properties of soils and laboratory methods for their determination. Moscow: Nedra, 1974. pp 182 - 191.

[7] Vatulyan A.O. Problems of identification of inhomogeneous properties of solids. Bulletin of the Samara state. University. 2007. Natural sciences. N 4. (54). pp.93-103.

[8] Frolova I.I., Andreev V.I. Thermal stress state of rock massif with a spherical cavity taking into account inhomogeneity of the medium. Series of books: MATEC Web of Conferences Volume 86, 2016. Article number: UNSP 03010. 
[9] Andreev V.I., Avershyev A.S. Moisture elasticity of an inhomogeneous thick-walled hollow sphere under non-stationary moisture conditions. Construction mechanics of engineering structures and structures (PFUR). №1. 2014. pp. 30-36.

[10] Zyuzin V.A. Axisymmetric problem of the theory of elasticity for a sphere under the radialpower law of the variation of elastic constants.

Interuniversity. Scientific. Coll., Issue 13, Dnepropetrovsk, 1971, pp. 89-93.

[11] A.A. Samarskiy, E.S. Nikolaev, Methods for solving grid equations. Nauka, Moscow, 1978. 